\title{
Ageostrophic Forcing in a Height Tendency Equation
}

\author{
ANTHONY R. LUPO \\ Department of Soil and Atmospheric Sciences, University of Missouri-Columbia, Columbia, Missouri
}

(Manuscript received 16 May 2001, in final form 29 June 2001)

\begin{abstract}
The role of the ageostrophic vorticity tendencies in the Zwack-Okossi vorticity tendency equation has been examined within the context that this term in the equation acts as a correction term for the vorticity tendency or height tendency calculations. In this paper, this term is shown to act as both a response to initial forcing and as a forcing mechanism itself in modulating the initial forcing. Using an earlier methodology for partitioning the wind field and the height field into their divergent and rotational parts, the ageostrophic wind is shown to contain components of both parts. Then, using an omega equation, an ageostrophic vorticity tendency is calculated, which has forcing mechanisms similar to a height tendency equation. This ageostrophic tendency is then used in this height tendency equation and provides for an improved method of calculating the ageostrophic wind rather than simply calculating it as a residual. Two case studies are then examined to demonstrate this point. For both case studies, the calculated height tendencies using the derived ageostrophic winds and those calculated as a residual overestimated the height tendencies, especially at the surface. While the correlation coefficients show that the two methods are comparable at $500 \mathrm{hPa}$, at the surface there is a notable degree of improvement in the pattern similarity. This resulted in an improved representation of the 500-hPa height tendencies for the onset of the Atlantic blocking event despite marginal improvements in the calculated field overall.
\end{abstract}

\section{Introduction}

Ageostrophic motions, and thus ageostrophic vorticities, have been traditionally viewed as a response to various dynamic and thermodynamic forcing processes, that when applied, perturb the balanced state of the atmosphere. Thus, ageostrophic motions have typically been analyzed in the context of an atmosphere that is initially in geostrophic or near geostrophic balance. Ageostrophic motions or ageostrophic winds have at least been considered to be a component or partition of the observed wind, that is the difference between the observed wind and the geostrophic wind (e.g., Trenberth and Chen 1988). The ageostrophic wind is proportional to and to the left (right) of the wind acceleration vector in the Northern (Southern) Hemisphere (e.g., Bluestein 1992). An atmosphere initially in geostrophic balance will respond to some imposed forcing mechanism that generates ageostrophic motions and induces vertical motions. Thus, vertical motions, and the "secondary" circulations they are part of, are also considered an important component in examining ageostrophic circulations (e.g., Uccellini et al. 1984; Keyser et al. 1989; Loughe et al. 1995).

Formulations for the ageostrophic winds have in-

Corresponding author address: Dr. Anthony R. Lupo, Department of Soil and Atmospheric Sciences, 109 Gentry Hall, University of Missouri-Columbia, Columbia, MO 65211.

E-mail: lupoa@missouri.edu cluded calculating this quantity as a residual, which then is dependent upon how geostrophy itself is defined (e.g., Phillips 1963; Blackburn 1985). Various alternative formulations for ageostrophic motions relating ageostrophic circulations to geostrophy have also been used in the examination of the three-dimensional circulations associated with upper-tropospheric jets, streaks, and fronts (see Trenberth and Chen 1988; Xu 1990, 1992; Keyser et al. 1989; Xu and Keyser 1993; Loughe et al. 1995). For the calculation of ageostrophic vorticity tendencies in a height tendency equation, such as the Zwack-Okossi (Z-O) equation (Zwack and Okossi 1986; Lupo et al. 1992), it would be ideal to find a relationship that yields a simple instantaneous estimate of the ageostrophic vorticity tendency that is a diagnostic quantity. However, to the author's knowledge, no such relationship exists.

Recently, several papers have been published which used the $\mathrm{Z}-\mathrm{O}$ equation as the diagnostic framework in the study of a variety of atmospheric phenomena. These phenomena include extratropical cyclones (e.g., Lupo et al. 1992; Rolfson and Smith 1996; Rausch and Smith 1996; Vasilj and Smith 1997; Zhang et al. 1999); midlatitude surface anticyclones (e.g., King et al. 1995); and blocking anticyclones (e.g., Uhl et al. 1992; Lupo and Smith 1995, 1998; Lupo 1997; Lupo and Bosart 1999). Of these papers, only Zwack and Okossi (1986), Lupo et al. (1992), and Rolfson and Smith (1996) discuss the physical processes represented by the forcing terms in a more detailed manner. Despite these discus- 
sions, however, only Lupo et al. (1992) addresses the role of ageostrophic vorticity tendencies since they used the generalized form of the $\mathrm{Z}-\mathrm{O}$ equation. Lupo et al. (1992) characterizes this term as a "correction term that accounts for the possibility that the unbalanced atmosphere may attempt to reach a balanced state other than geostrophic." Thus, ageostrophic vorticity tendencies, which appear explicitly in the Z-O equation, have not been viewed solely as either a forcing mechanism or a response to unbalanced forcing in a strict sense within this framework. Therefore, the exact role of the ageostrophic vorticities in this equation has not been satisfactorily addressed.

Then, the goal of this paper is to develop an expression that can be substituted for the ageostrophic vorticity tendency in the Z-O equation. This relationship is derived using the Trenberth and Chen (1988) methodology developed for describing the ageostrophic component of the wind. The partitioning procedure later used by Loughe et al. (1995) is similar to that used by Trenberth and Chen (1988). In deriving this relationship, it will be shown that the ageostrophic vorticity tendency term in the $\mathrm{Z}-\mathrm{O}$ equation can thus be interpreted in the traditional sense and as a forcing mechanism. Also, it will be shown that the ageostrophic vorticity tendency can at least be partially partitioned among the other terms in the $\mathrm{Z}-\mathrm{O}$ equation. This partitioning methodology is similar to that of Raisanen (1997) who carried out a partition of the stability term in the height tendency equation using the generalized omega equation. Finally, it will be shown that the derived relationship produces a better estimate of the total height tendencies derived from $\mathrm{Z}-\mathrm{O}$ height tendencies than simply calculating the ageostrophic vorticity tendency as a residual.

\section{The Zwack-Okossi equation}

The generalized $\mathrm{Z}-\mathrm{O}$ equation, which is a vorticity tendency equation derived by coupling the vorticity equation and the thermodynamic equation through the hydrostatic thickness tendency equation, is given by

$$
\begin{aligned}
\frac{\partial \zeta_{\mathrm{gL}}}{\partial t}= & \frac{1}{\left(p_{L}-p_{t}\right)} \int_{p_{t}}^{p_{L}}\left\{\left[-\mathbf{V} \cdot \nabla \zeta_{a}-\omega \frac{\partial \zeta_{a}}{\partial p}+\zeta_{a} \frac{\partial \omega}{\partial p}-\hat{\mathbf{k}}\left(\boldsymbol{\nabla} \omega \times \frac{\partial \mathbf{V}}{\partial p}\right)+\hat{\mathbf{k}} \cdot(\boldsymbol{\nabla} \times \mathbf{F})-\frac{\partial \zeta_{\mathrm{ag}}}{\partial t}\right]\right\} d p \\
& -\frac{1}{\left(p_{L}-p_{t}\right)} \frac{R}{f} \int_{p_{t}}^{p_{L}} \int_{p}^{p_{L}} \nabla^{2}\left(-\mathbf{V} \cdot \boldsymbol{\nabla} T+S \omega+\frac{\dot{Q}}{c_{p}}\right) \frac{d p}{p} d p,
\end{aligned}
$$

where $\zeta_{g}$ is the geostrophic relative vorticity and $p_{L}$ represents the near-surface level (the first 50-hPa-level above the earth's surface at any grid point). In (1), $\mathbf{V}$ is the horizontal wind vector, $Q$ the diabatic heating rate, $S$ the static stability parameter $[-(T / \theta)(\partial \theta / \partial p)], \omega$ the vertical motion $(d p / d t), \mathbf{F}$ the frictional force, $\zeta_{a}$ the absolute vorticity, and $\zeta_{\text {ag }}$ the ageostrophic vorticity, and $\boldsymbol{\nabla}$ the horizontal del operator on an isobaric surface. $R$, $c_{p}, f, T$, and $\theta$ are the gas constant for dry air, the specific heat at a constant pressure, the Coriolis parameter, absolute temperature, and the potential temperature, respectively. Also, the subscript " $p_{t}$ " is specified as the pressure at some sufficiently high pressure level chosen to encompass most of the atmospheric mass $(50 \mathrm{hPa}$ in this study). A more rigorous derivation can be found in Lupo et al. (1992).

This equation is similar to the Petterssen-Sutcliffe (PS) equation (Petterssen 1956, 320-325), which diagnoses surface development as a function of atmospheric forcing processes occurring between the surface and the nondivergent level, coupling these processes via the thermal wind. However, the $\mathrm{Z}-\mathrm{O}$ equation extends these concepts in order to expose more completely the importance of upper-tropospheric forcing on surface development. This equation is also versatile in that diagnoses at pressure levels other than the surface can be accomplished simply by solving the geostrophic vorticity tendency equation [see Eq. (3) in Lupo et al. 1992] for some specified level, as was done in Lupo and Smith (1995). The result is the $\mathrm{Z}-\mathrm{O}$ equation for pressure levels aloft in which the diagnostic quantity includes the near-surface geostrophic vorticity tendency as a forcing process [Eq. (1) in Lupo and Smith (1995)]

$$
\begin{aligned}
\left.\frac{\partial \zeta_{g}}{\partial t}\right|_{p_{i}}= & \left.\frac{\partial \zeta_{g}}{\partial t}\right|_{p_{L}} \\
& +\frac{R}{f} \int_{p_{i}}^{p_{L}} \nabla^{2}\left(-\mathbf{V} \cdot \boldsymbol{\nabla} T+S \omega+\frac{\dot{Q}}{c_{p}}\right) \frac{d p}{p}
\end{aligned}
$$

Equation (1) is then substituted for the near-surface geostrophic vorticity tendency on the right-hand side of (2). In Eq. (1), the diabatic heating and other quantities that must be parameterized are treated in more detail by Lupo et al. (1992), Lupo and Smith (1995), Rausch and Smith (1996), or Rolfson and Smith (1996). The vorticity tendencies are then relaxed to get height tendencies using sequential overrelaxation (Haltiner and Williams 1980). Height tendencies were examined since the geostrophic vorticities (and vorticity tendencies) tend to be weaker in the central region of large-scale phenomena, such as blocking anticyclones. Also, there is often 
more than one center of strong geostrophic vorticity (or vorticity) tendency located along the periphery of the height center (e.g., Sinclair 1996).

Another important advantage to using the $\mathrm{Z}-\mathrm{O}$ equation is that observed quantities can be used in (1) instead of a geostrophic estimation (Zwack and Okossi 1986). Since the observed winds, and thus vorticity tendencies, can be partitioned mathematically into both geostrophic and ageostrophic components, as was done in the derivation of the Z-O equation (see Lupo et al. 1992), this gives rise to an ageostrophic vorticity tendency term in Eq. (1). Lupo et al. (1992) describe the manner in which all the terms in Eq. (1) physically force vorticity and height changes. With the exception of the ageostrophic vorticity tendency term, those arguments are appropriate to the extent that the atmosphere attempts to achieve some geostrophically balanced state. If the atmosphere does not reach a new geostrophic equilibrium within the timescale defined by both the dataset used and the phenomenon studied, then not all of the vorticity forcing is being utilized to produce geostrophic vorticity changes. Rather, a portion is being utilized to produce ageostrophic vorticity changes. Thus, the ageostrophic vorticity tendency term provides an "adjustment" that corrects for this situation. Using the above argument, this term could then be considered as a response to atmospheric forcing. However, the appearance of this term on the right-hand side of (1) suggests that it can be expressed in terms of forcing mechanisms, and the following sections will demonstrate the validity of this interpretation.

\section{Calculating ageostrophic vorticity tendencies}

As described in the previous section, the ageostrophic vorticity tendency term represents an adjustment process in which this term corrects for the fact that the atmosphere may tend toward some new geostrophically balanced state or a balanced state that is other than geostrophic (Lupo et al. 1992; Rausch and Smith 1996). In order to derive a relationship for this term, the methodology of Trenberth and Chen (1988) is used. Trenberth and Chen (1988) argue that since the geostrophic wind relationship is a diagnostic relationship, the geostrophic wind could be inverted in order to define the geostrophic geopotential field. This field is defined by Trenberth and Chen (1988) as the geopotential field that, given the wind field, satisfies the geostrophic relation. Thus, the ageostrophic geopotential would be defined as the difference between the observed geopotential field and the geostrophic geopotential. They argue that in order to properly evaluate ageostrophic processes both the ageostrophic components of the wind and the geopotential should be considered (see Trenberth and Chen 1988 and references therein for a more detailed rationale).

Blackburn (1985) argues that the term ageostrophic is itself ambiguous since the geostrophic relationship could be calculated, for example, using an $f$-plane assumption (e.g., Phillips 1963), which he calls geostrophy-0 or "theoretician's geostrophy." Geostrophy can be defined such that $f$ varies [called geostrophy-1 by Blackburn (1985) or "synoptician's geostrophy"]. Each relationship can produce very different ageostrophic wind components if simply solved as a residual since geostrophy-1 contains divergence. Even so, in the use of geostrophy-1, the ageostrophic component does not account for all of the divergent component of the wind (e.g., Trenbeth and Chen 1988). Trenberth and Chen (1988) also show that the ageostrophic component of the wind contains a rotational component that, along with the divergent geopotential, can be quite substantial in the entrance and exit regions of jet maxima. Therefore, they demonstrate that it is useful to partition both the wind and geopotential height fields into divergent and rotational components in order to describe the ageostrophic component of the wind.

The following is a brief derivation of the Trenberth and Chen (1988) approach to calculating the ageostrophic wind component. For a more rigorous derivation, the reader should consult that paper. In addition to partitioning the wind into geostrophic and ageostrophic components, the wind can also be partitioned using Helmholtz theorem

$$
\begin{aligned}
\mathbf{V} & =\mathbf{V}_{\mathrm{g}}+\mathbf{V}_{\mathrm{ag}} \\
\mathbf{V} & =\mathbf{V}_{\mathrm{r}}+\mathbf{V}_{\mathrm{d}} \\
\mathbf{V}_{\mathrm{r}} & =\hat{\mathbf{k}} \times \boldsymbol{\nabla} \psi \\
\mathbf{V}_{\mathrm{d}} & =\boldsymbol{\nabla} \chi,
\end{aligned}
$$

where $\mathbf{V}_{\mathrm{g}}$ is the geostrophic wind (geostrophy-1 used here), $\mathbf{V}_{\text {ag }}^{\mathrm{g}}$ the ageostrophic component of the wind, $\mathbf{V}_{\mathrm{r}}$ the rotational component of the wind; $\mathbf{V}_{\mathrm{d}}$ the divergent component, and $\psi$ and $\chi$ are the geostrophic streamfunction and velocity potential, respectively.

However, a scalar field such as the geopotential can be partitioned using a balance relationship. Trenberth and Chen (1988) start with the appropriate form of the momentum equations corresponding to linear balance, and by taking the divergence of these equations, arrive at the linear balance equation:

$$
\nabla^{2} \Phi_{r}=\nabla \cdot f \nabla \psi
$$

provided that

$$
\begin{aligned}
& \nabla^{2} \Phi_{d}+u_{d} \beta-2 J\left(u_{r}, v_{r}\right) \\
& +\frac{1}{\cos \phi} \frac{\partial}{\partial y}\left[\frac{\left(u_{r}^{2}+v_{r}^{2}\right) \sin \phi}{a}\right]=0,
\end{aligned}
$$

where $J$ is the Jacobian operator and $\beta$ is $\partial f / \partial y$. By solving (4) and (5) the rotational $\left(\phi_{r}\right)$ and divergent $\left(\phi_{d}\right)$ geopotentials can be calculated. Expressions (4) and (5) are used here for consistency with Trenberth and Chen (1988) since the partition of $\Phi$ is not unique. While a 
higher-order balance model could be considered for use here (e.g., Lorenz 1960), the linear balance equation is simpler to use and more generally applicable (e.g., Trenberth and Chen 1988), especially for midlatitude synoptic-scale motions (e.g., Holton 1979). Trenberth and Chen (1988), however, solve for the rotational geopotential using (4), then solve for $\phi_{d}$ by subtracting $\phi_{r}$ from the total geopotential field. This procedure is replicated here. ply

Thus, using (3a), the ageostrophic component is sim-

$$
\mathbf{V}_{\mathrm{ag}}=\mathbf{V}-\mathbf{V}_{\mathrm{g}} .
$$

Then, partitioning $\mathbf{V}$ using (3b), (3c) and (3d), and $\mathbf{V}_{\mathrm{g}}$ using the height fields derived from (4) and (5), and substituting into (6) yields

$\mathbf{V}_{\mathrm{ag}}=\mathbf{k} \times \nabla \psi+\nabla \chi-\left(\frac{\hat{\mathbf{k}}}{f} \times \nabla \Phi_{r}+\frac{\hat{\mathbf{k}}}{f} \times \nabla \Phi_{d}\right)$,

where the rotational component of the wind can be equivalently expressed as

$$
\hat{\mathbf{k}} \times \boldsymbol{\nabla} \psi=\hat{\mathbf{k}} \times\left(\frac{1}{f} \nabla \Phi_{r}+\Phi_{r} \boldsymbol{\nabla} \frac{1}{f}\right) .
$$

By substituting (8) into (7), the expression for the ageostrophic wind component can be reduced to

$$
\mathbf{V}_{\mathrm{ag}}=\boldsymbol{\nabla} \chi-\left(\frac{\hat{\mathbf{k}}}{f} \times \nabla \Phi_{\mathrm{d}}\right)-\left(\frac{\beta \Phi_{r}}{f^{2}}\right) .
$$

This relationship for the ageostrophic component of the wind now accounts for all of the divergent component of the wind, the divergent geopotential, and the rotational component that cannot balance exactly owing to the variability of the Coriolis parameter $(f)$. The latter two come from the geostrophic component of the wind and are not accounted for by simply by calculating $\mathbf{V}_{\mathrm{ag}}$ as a residual. $\mathrm{Xu}$ and Keyser (1993) and Loughe et al. (1995) utilize similar relationships containing the divergent and rotational component of the ageostrophic wind. It should be noted here that the third term on the right-hand side would vanish if geostrophy-0 were used.

Then, $\chi$ can be calculated in (9) using the following relationships:

$$
\begin{aligned}
\nabla^{2} \chi & =\boldsymbol{\nabla} \cdot \mathbf{V} \quad \text { and } \\
\chi & =L_{i}\left(-\frac{\partial \omega}{\partial p}\right),
\end{aligned}
$$

where $\omega$ is vertical motion and $L_{i}$ is the inverse Laplacian. Hurrell and Vincent (1991) used this relationship to calculate the velocity potential $\chi$ and then used the first law of thermodynamics to calculate $\omega$.

Since vertical motions $(\omega)$ appear explicitly in the Z$O$ relationship, this variable must be estimated quantitatively. Here, a generalized form of the omega equation similar to that derived by Krishnamurti (1968) and utilized by Raisenen (1997) can be expressed as:

$$
\begin{aligned}
& \left(\nabla^{2} \sigma+f \zeta_{a} \frac{\partial^{2}}{\partial p^{2}}\right) \omega \\
& =-f \frac{\partial}{\partial p}\left[-\mathbf{V} \cdot \boldsymbol{\nabla} \zeta_{a}+\hat{\mathbf{k}} \cdot \boldsymbol{\nabla} \times \mathbf{F}\right. \\
& \left.-\left(\frac{\partial \mathbf{V}}{\partial p} \times \nabla \omega\right)-\frac{\partial \zeta_{\mathrm{ag}}}{\partial t}\right] \\
& +f \omega \frac{\partial^{2} \zeta}{\partial p^{2}}-\frac{R}{p} \nabla^{2}\left(-\mathbf{V} \cdot \nabla T+\frac{\dot{Q}}{c_{p}}\right),
\end{aligned}
$$

where $\sigma$ is the static stability parameter $[-(R / p)(T / \theta)$ $(\partial \theta / \partial p)]$. This form of the omega equation was chosen because of its compatibility with (1), that is, each of the forcing processes that appears in (1) has a complement in (11). In general, the effect of each term in (1) involves the creation of divergence/convergence fields as the result of ageostrophic motions imparted on balanced flow. As the mass field in a column of atmosphere adjusts to these new divergence/convergence fields in attempting to establish a new balanced state, the vertical motion profile must also adjust to the new atmospheric state. Thus, (1) and (11) represent complementary equations, and a similar equation for ageostrophic vorticity tendencies can be derived.

From Eq. (11), the vertical motions can be determined using sequential overrelaxation (Haltiner and Williams 1980) as subject to a suitable boundary condition, in this case the Dirichlet condition. Thus, (11) can be represented in symbolic form (e.g., Raisenen 1997)

$$
\begin{aligned}
\omega= & \omega_{\mathrm{hv}}+\omega_{\mathrm{f}}+\omega_{\mathrm{ti}}+\omega_{\mathrm{ag}}+\omega_{\mathrm{vv}}+\omega_{\mathrm{di}}+\omega_{\mathrm{ta}} \\
& +\omega_{\mathrm{diab}},
\end{aligned}
$$

where the subscripts correspond to each forcing mechanism. They are horizontal vorticity advection, friction, vorticity tilting, vertical vorticity advection, horizontal temperature advection, and diabatic processes, respectively. Thus, (10b) and (12) can be substituted into the first (velocity potential) term in (9) to obtain

$$
\begin{array}{r}
\mathbf{V}_{\mathrm{ag}}=\boldsymbol{\nabla}\left[L _ { i } \frac { \partial } { \partial p } \left(\omega_{\mathrm{hv}}+\omega_{\mathrm{f}}+\omega_{\mathrm{ti}}+\omega_{\mathrm{ag}}+\omega_{\mathrm{di}}+\omega_{\mathrm{ta}}\right.\right. \\
\left.\left.+\omega_{\text {diab }}\right)\right]-\frac{\hat{\mathbf{k}}}{f} \times \nabla \Phi_{d}-\frac{\beta \Phi_{r}}{f^{2}} .
\end{array}
$$

Thus, (13) becomes an expression for the ageostrophic wind containing forcing mechanisms that are compatible with those found in (1) and (11). No partioning of $\Phi_{d}$ was performed since it is not readily apparent that this can be done simply. Also, as stated earlier, this is calculated as a residual as in Trenberth and Chen (1988). Eq. (13) can now be used to calculate the ageostrophic vorticity tendency term in (1). Partitioning the first term 
in (13) among the forcing processes in (1) demonstrates mathematically the argument for the ageostrophic vorticity tendency term as a correction term (Lupo et al. 1992). Using the horizontal vorticity advection term as an example

$$
\left.\frac{\partial \zeta_{\mathrm{gL}}}{\partial t}\right|_{\mathrm{hv}}=-\frac{1}{p_{L}-p_{t}} \int_{p_{t}}^{p_{L}}-\mathbf{V} \cdot \nabla \zeta_{a}-\left.\frac{\partial \zeta_{\mathrm{ag}}}{\partial t}\right|_{\mathrm{hv}} .
$$

In section 2 , it was argued that the ageostrophic vorticity tendency could be considered a response to unbalanced forcing, provided that the atmosphere was initially in, or very close to, geostrophic balance. However, if the atmosphere is considered to be in an unbalanced or transitional state to begin with, then the time rate of change of that forcing mechanism can either modulate or enhance the "final" instantaneous geostrophic vorticity tendency by removing the ageostrophic component as in (13) or (14). Thus, the formulation also "eliminates" the expression for the ageostrophic vorticity tendency from the right-hand side of the equation, as many of the terms from the partitioned tendencies can be paired with the appropriate terms in (1). Additionally, as argued by Trenberth and Chen (1988), their partition of $\mathbf{V}_{\mathrm{ag}}$ is suitable for use in equations such as (11) and, thus presumably, its compliment, Eq. (1).

The ageostrophic motions produced by unbalanced forcing are proportional to the acceleration of the wind (e.g., Bluestein 1992). Typically, then the ageostropic vorticity tendencies are proportional to the magnitude of the forcing, that is, large forcing will result in larger geostrophic and ageostrophic vorticity tendencies (and typically of the same sign, aside from serendipitous horizontal and/or vertical distributions of the forcing functions [see Eq. (13), results in Lupo and Smith (1995), and Figs. 4d and $6 d$ here]. In (13) and (14), then, the ageostrophic tendency term, which is a correction term, would counter the ageostrophic component and the divergent part of the forcing functions in the $\mathrm{Z}-\mathrm{O}$ equation, which themselves could be partitioned in a similar manner to the development described in this section. Also, the time tendency of the rotational and divergent height tendencies act as correction terms and demonstrate that the geostrophic height tendency depends not only on the shape of the height tendency field, but its rate of change with time as well. Thus, as a correction term, the terms arising from the ageostrophic vorticity tendency can dampen the total forcing in (13), and as the forcing shrinks, the ageostrophic tendency term shrinks (and consequently ageostrophic forcing as well) until the system reestablishes geostrophic balance. This reestablishment of geostrophic balance likely represents a new balanced state rather than a return to the old state.

\section{Two case studies}

A comparison between the height tendencies calculated using (1) and (2), and by substituting expressions
(6) and (13) for the ageostrophic component of the wind, was then performed by examining two case studies. The first case is that of a blocking anticyclone using the height tendency fields associated with the life cycle of the blocking event studied in Lupo and Smith (1995), and a description of the event can be found there. This blocking event was a moderate-to-strong 5-day event that occurred over the middle and western Atlantic during late October and early November 1985. The second case study examines a rapidly developing cyclone event that occurred over the midwestern United States in a 24-h period between 0000 UTC 10 and 11 November 1998. This cyclone can be classified as a "land bomb" (central pressure fell $24 \mathrm{hPa}$ in $24 \mathrm{~h}$ ) as it developed very rapidly during the 0000 UTC to 1200 UTC time period (central pressure fell $13 \mathrm{hPa}$ ), and a brief synoptic description is given here.

At 1200 UTC 9 November, a weak surface disturbance was over southeastern Colorado, and strong surface fronts were analyzed across the southern Great Plains (warm front) and southwestern United States (cold front; Fig. 1). At $850 \mathrm{hPa}$, warm moist southerly flow was bringing in copious amounts of water vapor from over the Gulf of Mexico. The 500-hPa map (Fig. 1c) demonstrates that a strong trough was moving over the Rocky Mountain states during this time, and weak ridging could be found over the eastern part of the United States. At $300 \mathrm{hPa}$ a strong jet streak was digging into a trough over the western United States. By 0000 UTC 10 November, all the ingredients for a rapidly developing storm had moved into place, and the storm was under the poleward exit region of the jet streak. Strong convection was developing rapidly over eastcentral Kansas south into Oklahoma just prior to the 0000 UTC 10 November time period as indicated from satellite and Next Generation Weather Surveillance Radar-1988 Doppler (NEXRAD WSR-88D) imagery (not shown). Thus, latent heat release played a key role in cyclone development as shown in Fig. 2, which displays center point (of the surface low) surface height tendencies as calculated using (1) for 0000 UTC 10 November. Latent heat release is the third-largest height fall contributor to the total height tendencies (height falls).

\section{a. Data}

In the Lupo and Smith (1995) blocking event, the data used were analyses obtained from the National Aeronautics and Space Adminstration (NASA) Goddard Laboratory for Atmospheres (GLA; Schubert et al. 1993). These fields include the standard set of upperair parameters $u$ and $v$ (horizontal wind vectors in $\mathrm{m} \mathrm{s}^{-1}$ ), $z$ (height in $\mathrm{m}$ ), $T$ (absolute temperature), $\mathrm{RH}$ (relative humidity), and $q$ (mixing ratio in $\mathrm{g} \mathrm{kg}^{-1}$ ), which were interpolated linearly in $\ln (p)$ to $50-\mathrm{hPa}$ isobaric levels. Also, a suite of surface variables was provided with this dataset and the list of variables can be found in the reference cited above. The analyzed fields 

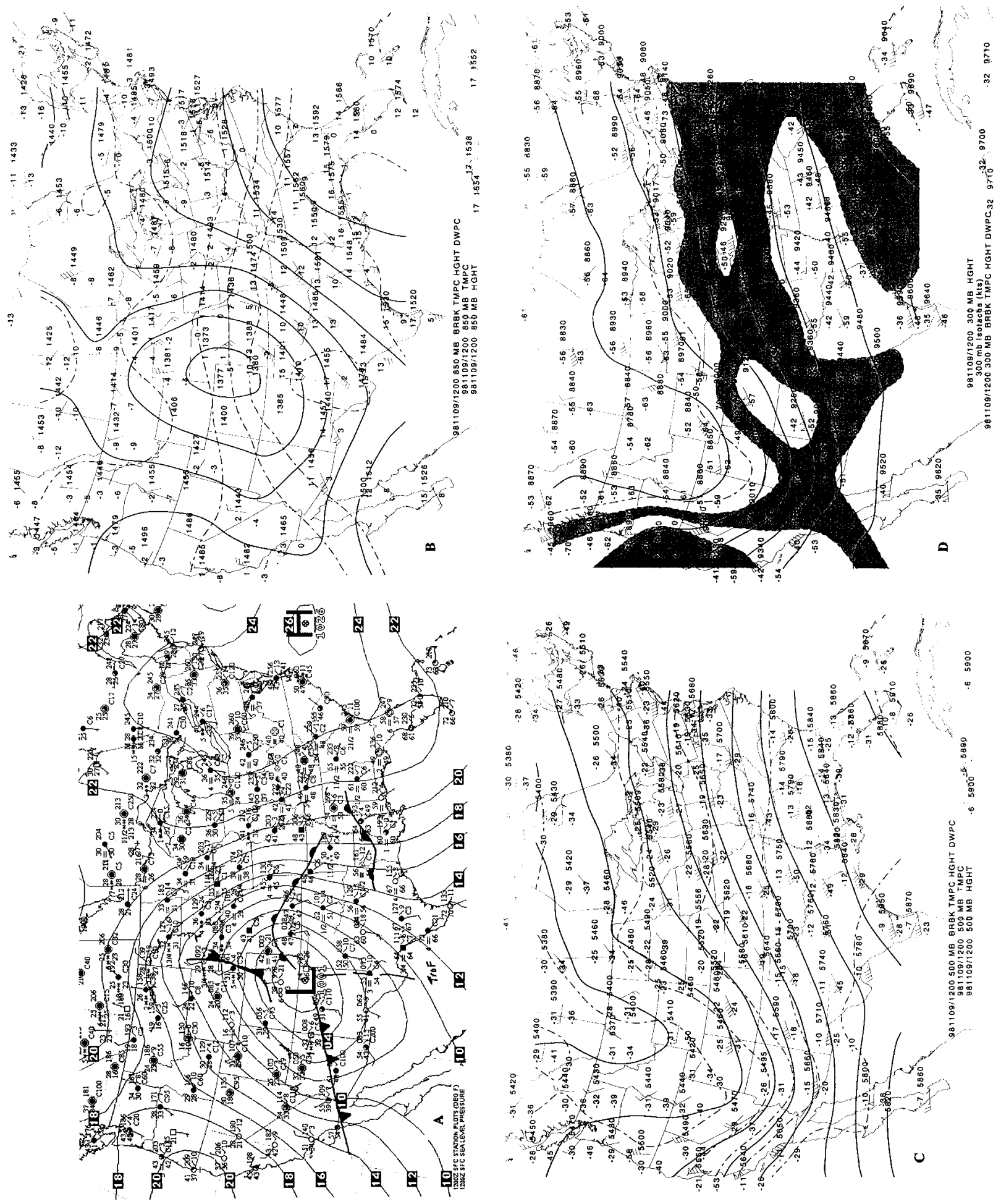
were provided on a $2.0^{\circ}$ latitude $\times 2.5^{\circ}$ longitude grid at 14 mandatory pressure levels from $1000 \mathrm{hPa}$ to 20 $\mathrm{hPa}$ at $6-\mathrm{h}$ intervals.

To study the 10-11 November 1998 midwestern cyclone, a different dataset was used. The dataset used in this study were the National Centers for Environmental Prediction (NCEP) gridded reanalyses, which are described in more detail by Kalnay et al. (1996). These analyses are archived at the National Center of Atmospheric Research (NCAR) and are available from the NCAR facilities in Boulder, Colorado. The NCEP gridded reanalyses used here were the upper-air analyses, quadratic in $\ln (p)$, interpolated onto 50-hPa isobaric levels. The horizontal resolution is $2.5^{\circ}$ latitude $\times 2.5^{\circ}$ longitude, with variables available at 6 -h intervals. The reanalyses are available on 17 mandatory levels from $1000 \mathrm{hPa}$ to $10 \mathrm{hPa}$, and include standard atmospheric variables such as geopotential height, temperature, relative humidity, vertical motion, $u$ and $v$ wind components, a set of various surface fields, and tropopause information. Lastly, since vertical motions are included in the NCEP reanalyses and not the Goddard Earth Observing System (GEOS-1) analyses, the vertical motions for both cases were calculated using (11) for experimental consistency.

\section{b. Results}

The resulting height tendencies were compared using the Trenberth and Chen (1988) ageostrophic winds (11) and the simple computation of the ageostrophic wind as a residual to calculate the ageostrophic tendency term in (1). The height tendencies compared for the blocking event were located within a moving $40^{\circ}$ latitude $\times 60^{\circ}$ longitude box centered on the blocking event itself. For the cyclone event, a box of the same size was centered on North America. Only three time periods were used in the case of the rapidly developing cyclone, and these were chosen to capture the cyclone development environment (1200 UTC 9 Nov to 1200 UTC 10 Nov. The height tendencies in each case were compared to height tendencies derived from the 24-h observed (centereddifference) geostrophic vorticity tendencies.

Tables 1 and 2 show the comparative statistics for the blocking event and the cyclone at both $500 \mathrm{hPa}$ and at the surface. Note that in both cases, there was a similar degree of improvement between the correlation coefficients for the surface fields, even though the correlations for the cyclone case were lower than the comparative statistic for the blocking event. At $500 \mathrm{hPa}$ there was little difference between the correlation coefficients for the calculation using the residual and the Trenberth and Chen (1988) method. As with the surface, the correlations are better for the height tendencies associated with the blocking event. Higher correlations for calculated height tendencies over a marine environment were also noted in Lupo et al. (1992). The higher correlations for the surface tendencies associated with the blocking event could partially be due to the elevation of the underlying surface. The observed tendencies in both cases were the 1000 -hPa surface, while the calculated height tendencies were the first 50-hPa level above the surface. Thus, in locations with elevated terrain, lower-tropospheric calculated height tendencies were being compared to observed height tendencies at the surface.

Generally, both methods overestimated the observed height tendencies, especially with the cyclone case. This overestimation was greater at the surface than aloft and for the cyclone rather than the blocking event. The overestimation in the cyclone case is not as severe if the comparison region used focuses more closely on the cyclone (a $20^{\circ}$ latitude $\times 40^{\circ}$ longitude box) and the overestimation for both the surface and 500 -hPa height tendencies using either method was by $50 \%$ or less (not shown). There was also an improvement (about 0.050.1 ) in the correlation coefficients when the smaller region was used, the improvement being larger for the surface fields. Since better pattern similarity would be an overriding concern for a diagnostic study, the Trenberth and Chen (1988) method of calculating the ageostrophic vorticity tendencies would be a superior technique. This improvement in pattern similarity occurs in spite of the fact that many more calculations were needed to get the same quantity, and thus, there was more chance for the introduction of numerical (e.g., truncation) error. The improvements in pattern similarity also occurred and were similar even though two different phenomena were examined in adjacent parts of the Northern Hemisphere and two different datasets were used with slightly different horizontal resolutions.

Figures 3-6 show the comparisons for each approach at the surface and at $500 \mathrm{hPa}$ to the observed height tendency results for the specific times used in Table 2. The most notable difference for the blocking case was over southern Greenland and extending out over the Atlantic where height tendencies using the residual calculation were much weaker than, or opposite in sign to, the height tendencies using the Trenberth and Chen (1988) ageostrophic winds (see Figs. 3 and 4). This region was near the exit region of a $300-\mathrm{hPa}$ jet (see

$\leftarrow$

FIG. 1. Synoptic maps showing the early development stage of the 10-11 Nov 1998 surface cyclone (1200 UTC 9 Nov) over the Midwest for the (a) surface, (b) 850-, (c) 500-, and (d) 300-hPa levels. The map (a) shows the surface pressure contoured every 4 hPa, and the fronts were drafted onto the figure from the original difax charts. The upper-air maps are standard height and temperature plots and use contour intervals of (b) 30 , (c) 60 , and (d) $120 \mathrm{~m}$, and $5^{\circ} \mathrm{C}$, respectively, for height and temperature. The wind speeds in (d) are shaded alternately light and dark and contoured every $20 \mathrm{kt}$ starting at $70 \mathrm{kt}$. 


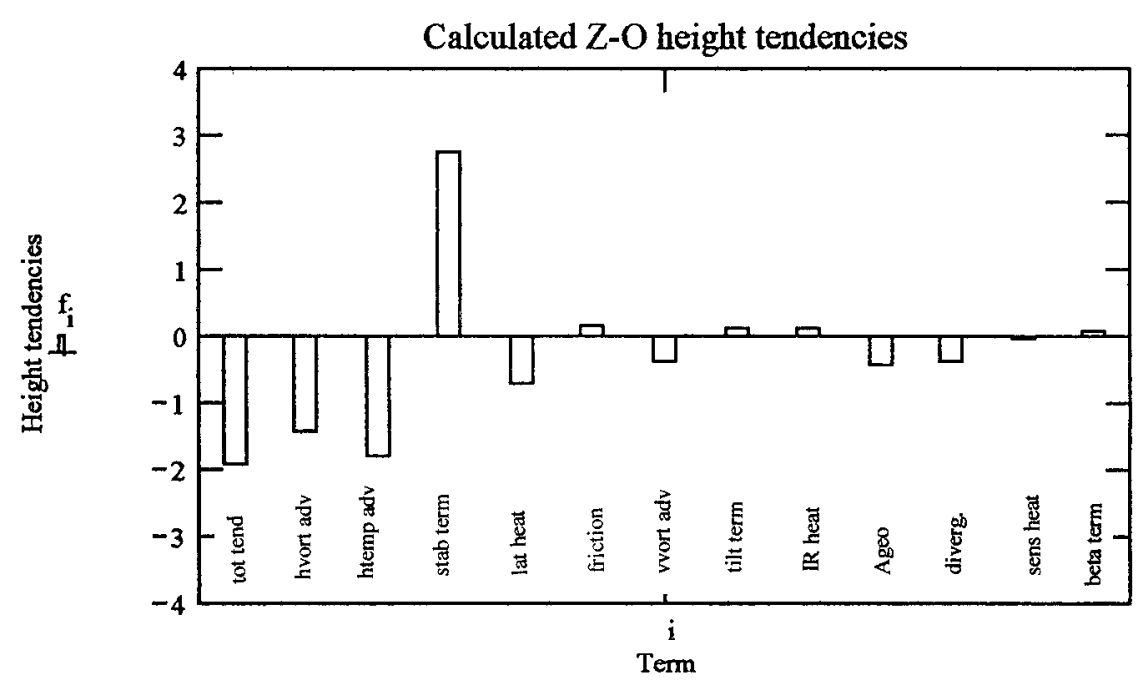

FIG. 2. Bar graph displaying averaged center point $\mathrm{Z}-\mathrm{O}$ calculated height tendencies $\left(\times 10^{-3}\right.$ $\left.\mathrm{m} \mathrm{s}^{-1}\right)$ for the cyclone over eastern Kansas and Nebraska $\left(37.5^{\circ} \mathrm{N}, 97.5^{\circ} \mathrm{W}\right)$ at 0000 UTC 10 Nov 1998.

Lupo and Smith 1995). These differences in the case of the block onset event at $500 \mathrm{hPa}$ lead one to interpret that the block center $(\mathrm{X})$ is well within the height rise region using the Trenberth and Chen (1988) method, while using the residual calculation the block center lies on the zero tendency line. The derived method compares more favorably to observations than the residual method (see Fig. 4).

For the cyclone case (Figs. 5 and 6), there was remarkable similarity in the $\mathrm{Z}-\mathrm{O}$ calculated height tendency fields using both methods in the main region of interest (the midwest United States), and both produced height tendencies that were similar to the observed. Differences in the two calculated fields were located away from the region of interest around the cyclone, and this is especially true across eastern and southeast Canada. The above similarity between the fields was stronger at the $500-\mathrm{hPa}$ level than at the surface. At the surface, the $\mathrm{Z}-\mathrm{O}$ height tendencies using the Trenberth and Chen

TABLE 1. Comparative statistics for calculating the total Z-O height tendencies using the ageostrophic winds calculated as a residual (R) and the Trenberth and Chen (1988: TC88) approach vs the observed finite difference height tendencies (OBS) for the Lupo and Smith (1995) blocking event. Displayed are correlation coefficients (CC), mean absolute values (MAV), and the ratio of the calculated and observed height tendencies displayed as a percentage (\% of OBS). Mean absolute values are given in units of $10^{-3} \mathrm{~m} \mathrm{~s}^{-1}$.

\begin{tabular}{|c|c|c|c|c|c|c|}
\hline & \multicolumn{3}{|c|}{ Entire Case } & \multicolumn{3}{|c|}{$\begin{array}{c}0600 \text { UTC } 30 \text { October } \\
1985\end{array}$} \\
\hline & $\mathrm{CCs}$ & MAVs & $\begin{array}{l}\% \text { of } \\
\text { OBS }\end{array}$ & $\mathrm{CCs}$ & MAVs & $\begin{array}{l}\% \text { of } \\
\text { OBS }\end{array}$ \\
\hline Surface $(\mathrm{R})$ & 0.76 & 1.25 & 150 & 0.69 & 1.29 & 153 \\
\hline (TC88) & 0.83 & 1.79 & 215 & 0.78 & 2.07 & 244 \\
\hline $500 \mathrm{hPa}(\mathrm{R})$ & 0.79 & 0.87 & 85 & 0.78 & 0.99 & 75 \\
\hline (TC88) & 0.78 & 1.29 & 126 & 0.80 & 1.77 & 134 \\
\hline
\end{tabular}

(1988) method place the maximum region of height falls in the Midwest closer to the observed height tendencies than height tendency calculation using the residual method. Despite the notable similarity between the calculated fields in the region of interest, there was still some improvement in the pattern similarity for the surface fields as shown in Table 2.

\section{Summary and conclusions}

In this study, the role of the ageostrophic vorticity tendencies in the $\mathrm{Z}-\mathrm{O}$ equation were examined in the context that this term acts as a correction term within the vorticity tendency or height tendency calculations. This term is shown theoretically to act as both a response to initial forcing and as a forcing mechanism itself in modulating the initial forcing. This study focuses on one particular term within the $\mathrm{Z}-\mathrm{O}$ equation and is similar to the study of Martin (1998) who examined the role of the deformation term in the omega equation. A partition of the wind fields using the methodology of Trenberth and Chen (1988) (and others following them) demonstrated that the ageostrophic winds contain the divergent component of the wind as well as components

TABLE 2. As in Table 1 except for the 10-11 Nov 1998 cyclone case.

\begin{tabular}{|c|c|c|c|c|c|c|}
\hline & \multicolumn{3}{|c|}{ Entire Case } & \multicolumn{3}{|c|}{$\begin{array}{c}0000 \text { UTC } 10 \text { Novembe } \\
1998\end{array}$} \\
\hline & $\mathrm{CCs}$ & MAVs & $\begin{array}{l}\% \text { of } \\
\text { OBS }\end{array}$ & $\mathrm{CCs}$ & MAVs & $\begin{array}{l}\% \text { of } \\
\text { OBS }\end{array}$ \\
\hline Surface (R) & 0.55 & 0.93 & 151 & 0.55 & 0.91 & 154 \\
\hline (TC88) & 0.59 & 1.40 & 226 & 0.57 & 1.36 & 231 \\
\hline $500 \mathrm{hPa}(\mathrm{R})$ & 0.76 & 1.09 & 130 & 0.76 & 1.11 & 139 \\
\hline (TC88) & 0.74 & 1.52 & 181 & 0.74 & 1.52 & 190 \\
\hline
\end{tabular}



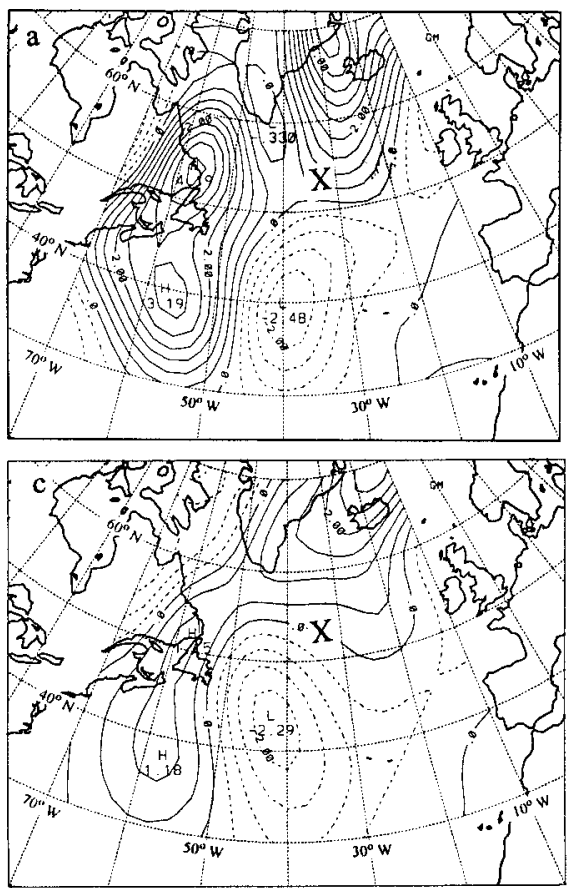
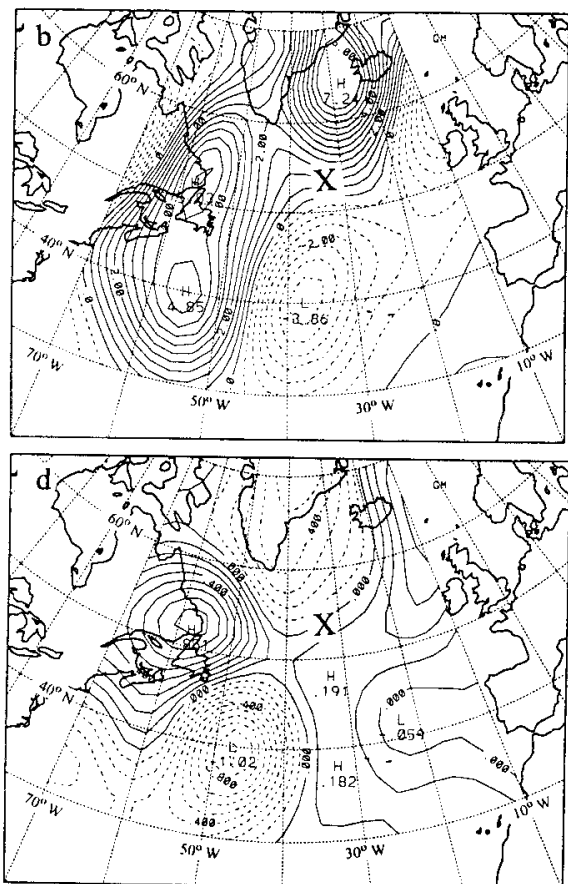

Fig. 3. Near-surface height tendency fields $\left(\times 10^{-3} \mathrm{~m} \mathrm{~s}^{-1}\right)$ calculated from the (a) total Z-O vorticity tendency using the residual ageostrophic winds, (b) the total Z-O vorticity tendency using the Trenberth and Chen (1988) ageostrophic winds, (c) the total finite-difference observed height tendency, and (d) the ageostrophic vorticity tendency term using the residual ageostrophic winds. The contour interval is 0.5 units.
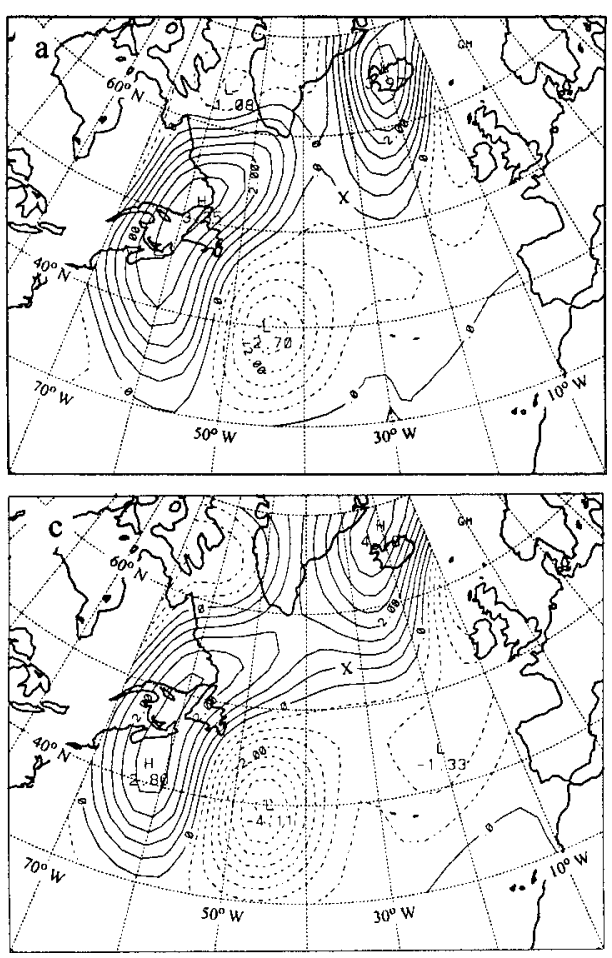
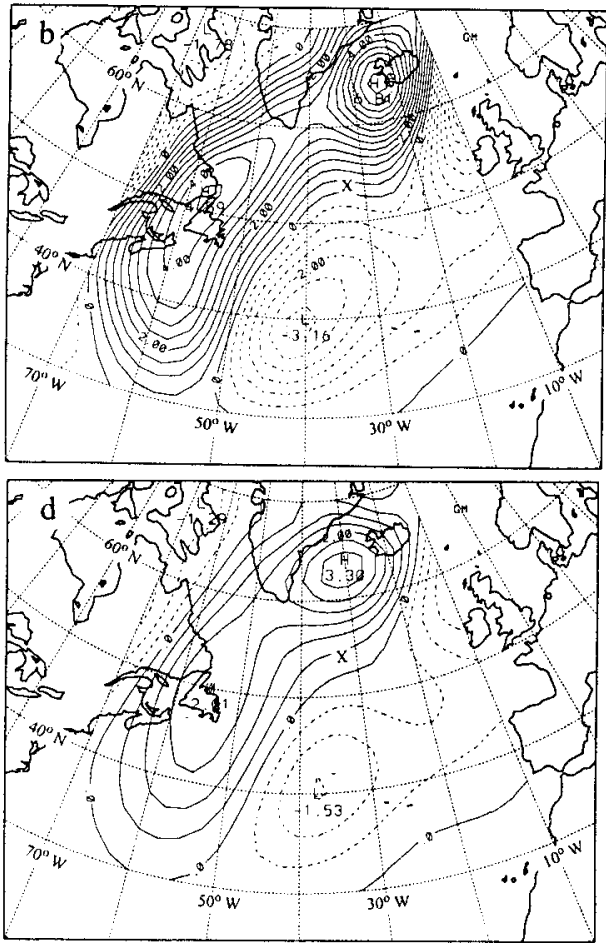

FIG. 4. As in Fig. 3 except at $500 \mathrm{hPa}$ and using the (d) Trenberth and Chen (1988) approach for calculating the ageostrophic winds. 

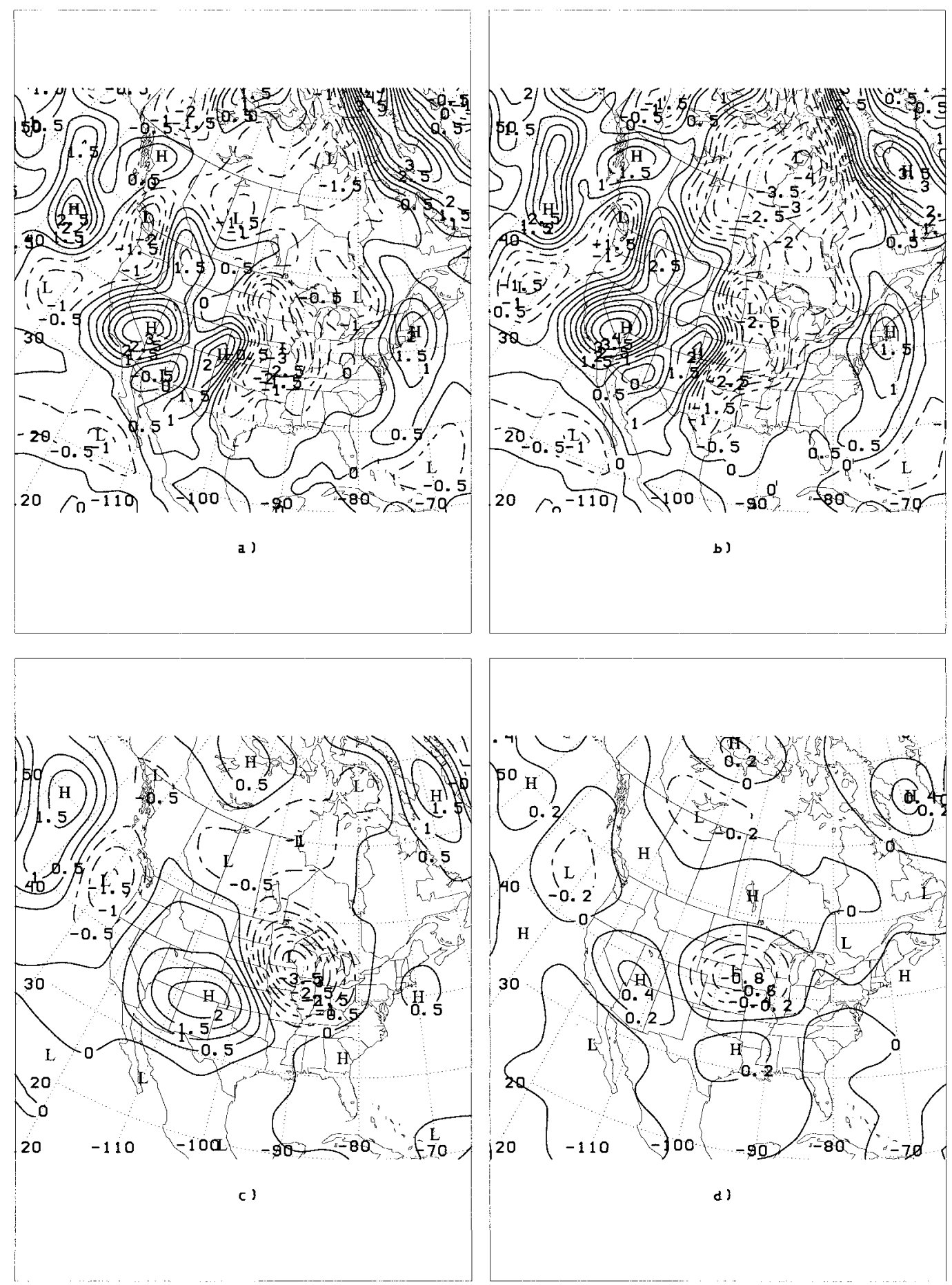

FIG. 5. As in Fig. 3 except for the cyclone case.

that resulted from the partition of the geostrophic wind (the geopotential field) into its divergent and rotational components. This partition demonstrates that an equation can be derived for the ageostrophic vorticity tendency that is complementary to the $\mathrm{Z}-\mathrm{O}$ equation and the omega equation. Each forcing mechanism in the Z-
$\mathrm{O}$ equation has an ageostrophic component that can be "corrected" for by subtracting out the ageostrophic forcing which can be partitioned in a similar manner to that of Raisanen (1997). Their study partitioned the vertical motion (stability) term in a height tendency equation using the omega equation. The strength of the 

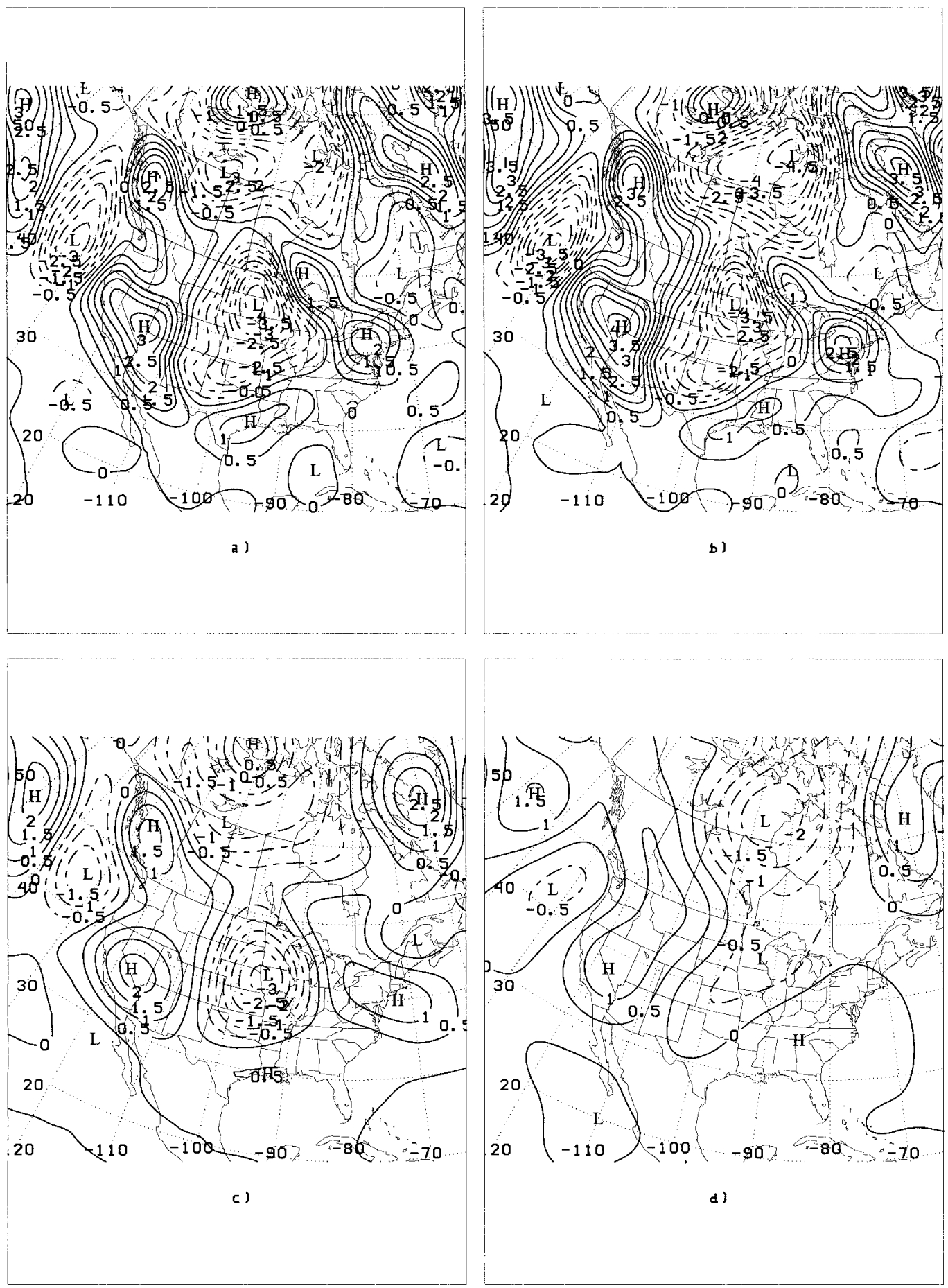

FIG. 6. As in Fig. 4 except for the cyclone case.

ageostrophic component is proportional to the time rate of change of the forcing itself, thus demonstrating the nonlinear nature of height changes as forced by dynamic or thermodynamic forcing mechanisms.

Two case studies were then examined to determine whether or not this method improved the final calculated
$\mathrm{Z}-\mathrm{O}$ height tendencies as compared to observed height tendencies. These case studies examined an Atlantic region blocking event and a rapidly developing North American cyclone. Even though two different phenomena were studied using two different datasets in two adjacent regions of the world, the conclusions were the 
same. The height tendencies using the derived method for calculating ageostrophic vorticity tendencies and the residual method both resulted in a comparable overestimation of the $500 \mathrm{hPa}$ and surface height tendencies. While the 500-hPa correlations to observed height tendencies were similar for both cases, the comparison of surface height tendencies resulted in improved pattern similarity using the derived ageostrophic winds. These improvements occur in spite of the larger number of calculations needed to compute the derived ageostrophic winds. With today's computing power, however, the time taken to calculate each was similar.

The most substantial improvement was the interpretation of the height tendency results for the onset of the blocking case. The block center was shown to be well within the area of height rises as calculated from the derived method as opposed to lying near the zero tendency line as with the residual ageostrophic calculation. The derived method compared more favorably to observations in this respect as well. For the cyclone case, the calculated $\mathrm{Z}-\mathrm{O}$ height tendency results within the area of interest using both methodologies were similar. There was some improvement in the pattern similarity, but these regions were outside the region of the cyclone.

Acknowledgments. The author would like to thank Dr. James T. Moore, Dr. Phillip J. Smith, Dr. Patrick Market, Dr. Peter Zwack, and the two anonymous reviewers for their helpful comments and insight at various stages of progress toward completion of this work. I would also like to thank Dr. Market for his assistance with constructing some of the figures.

\section{REFERENCES}

Blackburn, M., 1985: Interpretation of ageostrophic winds and implications for jet stream maintenance. J. Atmos. Sci., 42, 26042630.

Bluestein, H. B., 1992: Synoptic-Dynamic Meteorology in the Midlatitudes. Vol. I, Principles of Kinematics and Dynamics, Oxford University Press, $431 \mathrm{pp}$.

Haltiner, G. J., and R. T. Williams, 1980: Numerical Prediction and Dynamic Meteorology. John Wiley \& Sons, 478 pp.

Holton, J. R., 1979: An Introduction to Dynamic Meteorology. 2d ed. Academic Press, $391 \mathrm{pp}$.

Hurrell, J. W., and D. G. Vincent, 1991: On the maintenance of shortterm sub-tropical wind maxima in the Southern Hemisphere during SOP-1, FGGE. J. Climate, 4, 1009-1022.

Kalnay, E., and Coauthors, 1996: The NCEP/NCAR 40-Year Reanalysis Project. Bull. Amer. Meteor. Soc., 77, 437-471.

Keyser, D., B. D. Schmidt, and D. G. Duffy, 1989: A technique for representing three-dimensional vertical circulations in baroclinic disturbances. Mon. Wea. Rev., 117, 2463-2494.

King, M. L., P. J. Smith, and A. R. Lupo, 1995: A diagnosis of the development of a winter anticyclone over North America. Mon. Wea. Rev., 123, 2273-2284.

Krishnamurti, T. N., 1968: A diagnostic balance model for studies of weather systems of low and high latitudes, Rossby numbers less than one. Mon. Wea. Rev., 96, 518-530.
Lorenz, E. N., 1960: Energy and numerical weather prediction. Tellus, 12, 364-373.

Loughe, A. F., C. C. Lai, and D. Keyser, 1995: A technique for diagnosing three-dimensional ageostrophic circulations in baroclinic disturbances on limited area domains. Mon. Wea. Rev., 123, 1476-1504.

Lupo, A. R., 1997: A diagnosis of two blocking events that occurred simultaneously over the mid-latitude Northern Hemisphere. Mon. Wea. Rev., 125, 1801-1823.

_ , and P. J. Smith, 1995: Planetary and synoptic-scale interactions during the life-cycle of a mid-latitude blocking anticyclone over the North Atlantic. Tellus, 47A, 575-596.

$\longrightarrow$, and — 1998: The interactions between a midlatitude blocking anticyclone and a synoptic-scale cyclone occurring during the summer season. Mon. Wea. Rev., 126, 503-515.

, and L. F. Bosart, 1999: An analysis of a relatively rare case of continental blocking. Quart. J. Roy. Meteor. Soc., 125, 107-138.

_ , P. J. Smith, and P. Zwack, 1992: A diagnosis of the explosive development of two extratropical cyclones. Mon. Wea. Rev., 120, $1490-1523$

Martin, J. E., 1998: On the deformation term in the quasigeostrophic omega equation. Mon. Wea. Rev., 126, 2000-2007.

Petterssen, S., 1956: Weather Analysis and Forecasting. Vol. I, 2d ed. McGraw-Hill, 428 pp.

Phillips, N. A., 1963: Geostrophic motion. Rev. Geophys., 1, $123-$ 176.

Raisanen, J., 1997: Height tendency diagnostics using a generalized omega equation, the vorticity equation, and a nonlinear balance equation. Mon. Wea. Rev., 125, 1577-1597.

Rausch, R. L. M., and P. J. Smith, 1996: A diagnosis of a modelsimulated explosively developing extratropical cyclones. Mon. Wea. Rev., 124, 875-904.

Rolfson, D. M., and P. J. Smith, 1996: A composite study of synopticscale extratropical cyclone development over the United States. Mon. Wea. Rev., 124, 1084-1099.

Schubert, S. D., R. D. Rood, and J. Pfaendtner, 1993: An assimilated dataset for earth science applications. Bull. Amer. Meteor. Soc., 74, 2331-2342.

Sinclair, M. R., 1996. A climatology of anticyclones and blocking for the Southern Hemisphere. Mon. Wea. Rev. 124, 245-263.

Trenberth, K. E., and S.-C., Chen, 1988: Rotational and divergent geopotential components. J. Atmos. Sci., 45, 2949-2960.

Uccellini, L. W., P. J. Kocin, R. A. Petersen, C. H. Wash, and K. F Brill, 1984: The Presidents' Day cyclone of 18-19 February 1979: Synoptic overview and analysis of the subtropical jet streak influencing the pre-cyclogenetic period. Mon. Wea. Rev., $112,31-55$

Uhl, M. A., P. J. Smith, A. R. Lupo, and P. Zwack, 1992: The diagnosis of a pre-blocking explosively-developing extratropical cyclone system. Tellus, 44A, 236-251.

Vasilj, J. M., and P. J. Smith, 1997: A comparison of extended and quasigeostrophic dynamics for a case of small-Rossby number extratropical cyclone development. Mon. Wea. Rev., 125, 33473356.

Xu, Q., 1990: Cold and warm frontal circulations in an idealized moist semigeostrophic baroclinic wave. J. Atmos. Sci., 47, 23372352.

_ 1992: Ageostrophic pseudovorticity and geostrophic C-vector forcing: A new look at the $\mathrm{Q}$ vector in three dimensions. $J$. Atmos. Sci., 49, 981-990.

_, and D. Keyser, 1993: Barotropic and baroclinic ageostrophic winds and completeness of solution for the psi equations. $J$. Atmos. Sci., 50, 588-596.

Zhang, D. L., E. Radeva, and J. Gyakum, 1999: A family of frontal cyclones over the western Atlantic Ocean. Part II: Parameter studies. Mon. Wea. Rev., 127, 1745-1760.

Zwack, P., and B. Okossi, 1986: A new method for solving the quasigeostrophic omega equation by incorporating surface pressure tendency data. Mon. Wea. Rev., 114, 655-666. 